\title{
Model for Frequency Dependence of Thermal Permeability in Order to Quantify the Effects of Thermal Exchange on Wave Propagation in Multi Layered Porous Medium
}

\author{
Allen Teagle-Hernandez ${ }^{1,2}$, Ortwin Ohtmer ${ }^{1}$, Duyen Nguyen ${ }^{1}$ \\ ${ }^{1}$ Mecahnical and Aerospace Engineering Department, California State University, Long Beach, USA \\ ${ }^{2}$ Vibro-Acoustic Department, HSEA Tech. Inc., Nagoya, Japan \\ Email: allen.teagle@csulb.edu
}

How to cite this paper: Teagle-Hernandez, A., Ohtmer, O. and Nguyen, D. (2019) Model for Frequency Dependence of Thermal Permeability in Order to Quantify the Effects of Thermal Exchange on Wave Propagation in Multi Layered Porous Medium. Journal of Applied Mathematics and Physics, 7, 1440-1462.

https://doi.org/10.4236/jamp.2019.77097

Received: April 12, 2019

Accepted: July 3, 2019

Published: July 10, 2019

\begin{abstract}
The ability to quantify and predict the energy absorption/transmission characteristics of multi-layered porous medium is imperative if one is involved in the automotive, launch vehicle, commercial aircraft, architectural acoustics, petroleum exploration, or even in modeling human tissue. A case in point, the first four aforementioned fields rely on effective Noise and Vibration (NV) development for their commercial success. NV development requires the setting of NV targets at different system levels. The targets are then translated to Transmission Loss (TL), Insertion Loss (IL), and absorption (Alpha) performance for the multi-layered porous materials being utilized. Thus, it behooves to have a thorough understanding of the physics behind the energy dissipating mechanism of the material that entails the effects of the fluid meandering through the pores of the material and its interaction with the structural skeleton. In this section of the project the focus is on the thermal interchange that occurs within the porous medium. Via the acoustic modeling at the micro/macro level it is shown how this thermal exchange affects the acoustic compressibility within the porous material. In order to obtain a comprehensive approach the ensuing acoustic modeling includes the effects due to relaxation process, thus bulk viscosity and instantaneous entropy functions (effects due to vibration of diatomic molecules of air) are incorporated into the equation. The instantaneous entropy functions are explained by means of the Boltzmann's distribution, partition function, and quantum states. The concept of thermal length and its connection to thermal permeability is clarified. Lastly, the results for TL calculations employing the aforementioned thermal exchange into the Transfer Matrix Method with finite size
\end{abstract}


correction, (FTMM), pertaining to a simple multi-layered material is compared with experimentally obtained data.

\section{Keywords}

Porous Medium, Fluid Structure Interaction, Thermal Permeability, Thermal Length, Partition Function, Quantum Mechanics, Asymptotic Expansion, ket/bra Vectors

\section{Introduction}

Teagle et al. [1] derived a coupled set of fluid/structure equations for a porous medium applying asymptotic and homogenization techniques. Via this modeling it is established in [1] that there are mainly 3 modes of energy transformation: 1) The first mode is through the connection between the micro and macro structural framework of the porous skeleton, 2) the second is via the viscous boundary layer, and 3) the third interaction is through thermal (entropy) boundary layer. The combination of the viscous boundary layer and how tortuous the porous material is, results in the encapsulation of the fluid medium. This, in turn, changes the apparent mass of the structural medium. Details pertaining to these encapsulating phenomena can be found in the work by Johnson, et al. [2]. In [2] the concepts of tortuosity, viscous length, and viscous permeability are explained.

This current work concentrates in the third interaction mode. There is a boundary layer where the flow experiences changes in entropy, therefore a loss/gain of energy due to thermal exchange will occur. This thermal exchange also changes the acoustic bulk modulus at certain frequencies and in turn changing the speed of sound. This is depicted through Equation (31) found in [1]

$$
-i \omega^{*} p_{o}^{*}\left(\gamma+(\gamma-1) \operatorname{Pr} k^{*} i \omega^{*}\right)=-\nabla_{y}^{*} \cdot \mathbf{v}_{1}^{*}+i \omega^{*} \nabla_{x}^{*} \cdot \mathbf{u}_{o}^{*}+i \omega^{*} \nabla_{x}^{*} \cdot \mathbf{w}^{*}(\mathbf{x}, \mathbf{y}) \text { in } D_{f}(1)
$$

From this equation it is understood that the compressibility factor, $\beta_{a}$, is defined as

$$
\beta_{a}=\gamma+(\gamma-1) i \omega \frac{P r}{\mu \phi} \rho k^{\prime}
$$

where $\kappa_{a}=\frac{1}{\beta_{a}}$ is the bulk modulus of the air inside the pore. $k^{\prime}$ is the thermal permeability and is defined as

$$
\phi T_{o}=-\frac{k^{\prime}(\omega)}{\kappa} \frac{\partial P_{o}}{\partial t}
$$

$T_{o}, P_{o}$ are the first terms of the asymptotic expansion (macro scale) pertaining to the temperature and pressure respectively, their equations are derived in [1]. The bulk of this work is to derive a frequency dependent general expression for 
the thermal permeability, $k^{\prime}(\omega)$. In order to attain this goal a good understanding of the physics behind the thermal exchange process has to be accomplished. This is achieved by: 1) analyzing the intensity escaping the main acoustic mode or 2) deriving a partial differential equation incorporating the porous wall impedance due to the thermal/viscous boundary layer; both are accomplished here.

This work also models an additional energy absorbing phenomena and that is the relaxation process. This is the hysteresis effect due to compression/decompresssion cycle of the molecules. The application of quantum mechanics, explained via the construct of Hilbert Spaces along with its dual space, facilitates the modeling of this hysteresis effect.

The main interest of the current authors is to calculate transmission loss (TL), Insertion loss (IL), and absorption coefficient (Alpha) for multi-layered porous medium. Therefore, calculation results for TL pertaining to a layered material, consisting of 2 different foams, are shown. This calculation is achieved by incorporating all of the aforementioned energy phenomena into the Finite-Correction Transfer Matrix Method (FTMM) [3] [4] [5].

In addition to the goals mentioned in the last paragraph a parallel effort is underway to lay down the mathematical foundation to understand how the propagating energy transfigures as it travels through the porous medium. This creates a tool that will allow design teams to make efficient absorption comparison of porous mediums consisting of different rheological substances.

\section{Formulation}

\subsection{Basic Statistical Mechanics: Partition Function/Boltzmann Distribution/Quantum States}

One of the goals is to obtain a comprehensive set of fluid equations that incorporates any phenomena that leads to energy depletion. This means that in addition to viscous and thermal effects that transpire within their respective boundary layers, a quantification of the hysteresis effects due to the delay in thermal response should be obtained and incorporated. The most efficient tool to describe this process and for that matter any process is to apply statistical mechanic techniques. Once the Partition Function, $Z$, is obtained any macro level characteristic pertaining to the system can be derived. In this study Boltzmann's version of the combinatorial arrangement, $\tilde{C}=\frac{N !}{\prod_{i-1}^{m} n_{i} !}$, is used. $N$ represents the number of states, and $n_{i}$ are the number of states at each energy level. Applying Sterling's formula, this arrangement number becomes,

$C=\ln (\tilde{C})=-\sum_{i} p_{i} \ln \left(p_{i}\right)$, where $p_{i}=\frac{n_{i}}{N}$, is the probability of being in the $\mathrm{i}^{\text {th }}$ energy state that has energy $E_{i}$. The statistical mechanic definition of Entropy, $s$, is the measure of information plus its distribution and is defined as finding the $p_{i} s$ that will maximize the arrangement number, $C$, under the following con- 
straints: $\sum_{i} p_{i}=1$, and $\sum_{i} p_{i} E_{i}=E$ ( $E=$ Average Energy). From this mathematical framework, the temperature, $\bar{T}$, plays the role of a Lagrange Multiplier. The main results are that

$$
p_{i}=\frac{e^{-\beta E_{i}}}{Z}
$$

where $Z$ is the partition function defined as

$$
Z=\sum_{i} e^{-\beta E_{i}} \quad \beta=\frac{1}{\overline{\bar{T}}} \quad \bar{T}=k T \quad k=\text { Boltzmann Constant }
$$

and note that the units for $\bar{T}$ ("theorist" temperature) is in Joules. It is easily shown that the average energy and the Helmholtz Free Energy, $A$, are represented respectively as,

$$
E=-\frac{\partial \ln (Z)}{\partial \beta} \text { and } A=E-T S=-\bar{T} \ln (Z)
$$

In quantum mechanics (see Section 2.2 below) the probability density is defined as $p(r, t)=\psi \bar{\psi}=|\psi|^{2}$, where $\psi$ is a state vector of the system. Applying Schrodinger's equation, $i \hbar \frac{\partial \Psi}{\partial t}=\hat{H} \Psi(r, t)$, and Hamilton's operator, defined in Equation (19a), the following differential equation is derived which expresses the propagation of probability

$$
\frac{\partial p}{\partial t}+\nabla J=0, \quad J=\frac{i \hbar}{2 m}[\psi \nabla \bar{\psi}-\overline{\psi \nabla \psi]}
$$

$J$ is the probability flux. For adiabatic processes the divergence of the probability flux is shown to be zero, $\nabla J=0$; therefore the probability density is stationary with respect to time. This means that the $p_{i} s$ are adiabatic invariant (which in turn means that entropy is constant) and thus the following expression for pressure holds

$$
\begin{aligned}
& \left.P \stackrel{\text { adiabatic }}{\longrightarrow} \frac{\partial E}{\partial V}\right|_{S}=-\left.\frac{\partial E}{\partial V}\right|_{T}+\left.\left.\frac{\partial S}{\partial V}\right|_{T} \frac{\partial E}{\partial S}\right|_{V}=-\left.\frac{\partial E}{\partial V}\right|_{T}+\left.\frac{\partial S}{\partial V}\right|_{T} T \\
& =-\left.\frac{\partial(E-T S)}{\partial V}\right|_{T}=\left.\frac{T \partial \ln (Z)}{\partial V}\right|_{T}
\end{aligned}
$$

The average potential between the particles is defined as $U_{0}$ and under classical mechanic conditions the partition function is

$$
\begin{aligned}
& \qquad Z=Z_{0}(\beta)\left[1-\frac{\beta N^{2} U_{0}}{2 V}\right] \\
& Z_{0}(\beta)=\left(\frac{e}{\rho}\right)^{N}\left(\frac{2 m \pi}{\beta}\right)^{\frac{3 N}{2}} \text { is the partition function pertaining to the Ideas Gas. }
\end{aligned}
$$

In order to obtain a general expression for the average energy of the gas, Equation (6) is applied

$$
E=-\frac{\partial \ln (Z)}{\partial \beta}=N \frac{3}{2} \bar{T}+\frac{N^{2}}{2 V} U_{0}=\left[\frac{3}{2} \bar{T}+\frac{\tilde{\rho}}{2} U_{0}\right] N
$$


Diatomic molecules like $\mathrm{O}_{2}$ and $\mathrm{N}_{2}$ can be modeled as stiff oscillators. As a simple representative example, a model of a simple oscillator with stiffness $k$ will be studied. The total energy for this system is

$$
E_{T}=\frac{p^{2}}{2 m}+\frac{k x^{2}}{2}
$$

Via the Boltzmann distribution, the Partition Function pertaining to the oscillator, $Z_{o s}$, is

$$
Z_{o s}=\iint e^{-\beta \frac{p^{2}}{2 m}} e^{\frac{k x^{2}}{2}} d p d x=\int e^{-\beta \frac{p^{2}}{2 m}} d p \int e^{\frac{k x^{2}}{2}} d x
$$

By applying Gaussian integrals the Partition Function for an undamped oscillator is

$$
Z_{o s}=\sqrt{\frac{2 m \pi}{\beta}} \sqrt{\frac{2 \pi}{\beta k}}=\frac{2 \pi}{\beta} \sqrt{\frac{m}{k}}=\frac{2 \pi}{\omega_{n}} \frac{1}{\beta}
$$

As before, Equation (6) is used in order to obtain the average energy of the oscillating system

$$
-\frac{\partial \ln \left(Z_{o s}\right)}{\partial \beta}=-\frac{\partial\left[\ln \left(\frac{2 \pi}{\omega_{n}}\right)-\ln (\beta)\right]}{\partial \beta}=\frac{1}{\beta}=\bar{T}
$$

The analysis above, especially Equation (11), assumes classical mechanic conditions. Equation (13) states that the average energy of the oscillating system is $\bar{T}$ which, based on prior analysis, is connected to kinetic energy. This result is somewhat suspect since it states that regardless of how stiff the system is, the average energy is always $\bar{T}$. In classical mechanics, the path integrals measured with respect to the Max Planck constant, $\hbar$, are relatively large. An observable in classical mechanics is simply a real function in Euclidean space. In quantum mechanics every possible state of a given system corresponds to a separable Hilbert Space over the complex number field. Additionally, to every physical observable there corresponds, in the Hilbert space, a linear Hermitian operator that has a complete set of orthogonal eigenvectors. The following limit applies to systems oscillating at relatively high energy levels,

$\lim _{n \rightarrow \infty} \frac{\Delta E}{E_{n}}=\lim _{n \rightarrow \infty} \hbar \omega /\left[\left(n+\frac{1}{2}\right) \hbar \omega\right] \rightarrow 0$ thus, in classical mechanics, it can be assumed that the energy forms a continuous spectrum; as compared to quantum mechanics where there is now a quantization (discretization) of the energy states [6]. In quantum mechanics a unit of energy is proportional to the natural frequency of the system by the constant, $\hbar$. The $\mathrm{n}^{\text {th }}$ energy state is defined as

$$
E_{n}=n \hbar \omega
$$

Thus, the Partition Function is

$$
Z_{q}=\sum_{n} e^{-\beta n \hbar \omega}=\sum_{n}\left(e^{-\beta \hbar \omega}\right)^{n}=\frac{1}{1-e^{-\beta \hbar \omega}}
$$

Equation (6) is utilized once more in order to obtain the average energy of the 
stiff oscillator

$$
E=-\frac{\partial \ln (Z)}{\partial \beta}=-\frac{1}{Z} \frac{\partial Z}{\partial \beta}=\frac{\hbar \omega e^{-\beta \hbar \omega}}{1-e^{-\beta \hbar \omega}}
$$

At high temperatures $\beta \ll 1$ and therefore Equation (15) becomes

$$
E=\frac{\hbar \omega e^{-\beta \hbar \omega}}{1-e^{-\beta \hbar \omega}} \sim \frac{\hbar \omega}{\beta \hbar \omega}=\frac{1}{\beta}=\bar{T}
$$

Therefore at high temperature levels there is enough energy such that the quantization of energy is not required. The criteria to follow are the following

$$
\beta \hbar \omega>1 \rightarrow \text { Quantum Mechanics } \quad \beta \hbar \omega<1 \rightarrow \text { Classical Mechanics }
$$

The cross-over point is $\hbar \omega=\bar{T}$. Lastly, it is possible to extract the Energy's standard deviation from the Partition Function. It is easy to show that $\left\langle E^{2}\right\rangle=\frac{1}{Z} \frac{\partial^{2} Z}{\partial \beta^{2}}$. What follows is the following definition

$$
\left\langle(\Delta E)^{2}\right\rangle=\left\langle E^{2}\right\rangle-\langle E\rangle^{2}=\frac{1}{Z} \frac{\partial^{2} Z}{\partial \beta^{2}}-\frac{1}{Z^{2}}\left(\frac{\partial Z}{\partial \beta}\right)^{2}=\frac{\partial^{2} \ln (Z)}{\partial \beta^{2}}=-\frac{\partial E}{\partial \beta}=-\frac{\partial E}{\partial T} \frac{\partial \bar{T}}{\partial \beta}(18 \mathrm{a})
$$

Recognize that $\frac{\partial E}{\partial \bar{T}}=C_{V}$, the specific heat constant at constant volume and $\frac{\partial \bar{T}}{\partial \beta}=-\bar{T}^{2}$

$\left\langle(\Delta E)^{2}\right\rangle=\bar{T}^{2} C_{V}$ or $\langle\Delta E\rangle=\bar{T} \sqrt{C_{V}}$ fluctuation-susceptibility Theorem (18b)

The specific heat constant at constant volume is proportional to standard deviation of the energy.

\subsection{Relaxation Process: Fluid Hysteresis/Bra-Ket Vectors, Schrodinger's Equation}

The vibration due to the collision of molecules extract energy from the passing wave but releases the energy after some delay, thus the relaxation process. This delay, akin to mechanical damping systems leads to hysteretic energy losses. The internal energy can be partitioned into translated (defined relative to the average flow velocity), rotational kinetic energy and energy due to molecular natural frequency,

$$
u=u_{t r}+u_{r o t}+\sum_{v} u_{v}
$$

$u_{v}$ quantum energy level/mass

The temperatures, $T_{t r}$ and $T_{r o t}$ related to translational and rotational energies are larger than the temperatures related to molecular oscillation. They usually satisfy the second criteria in Equation (17) and therefore can be considered using Classical Theory. For the molecular vibration case, $\hbar \omega>k T_{v}$, and, based on Equation (17), quantum mechanics should be applied.

The first postulate in quantum theory states that everything that can be known about the state of a system can be extracted from its state vector (wave function) and this is represented as a vector in Hilbert Space; these are denoted 
as ket-vectors $|\Psi(x)\rangle$. The bra-vectors are denoted as $\langle\Psi(x)|$, the vector space of bras is the dual space(one-to-one correspondence with the space of functionals) to the kets vector space. The Riesz representation theorem allows the following notation $\langle\Psi \mid \Psi\rangle$.

The 1-D Hamilton operator is given as

$$
\hat{H}=\frac{\hat{p}^{2}}{2 m}+V(\hat{x})=-\frac{h^{2}}{2 m} \frac{\partial^{2}}{\partial x^{2}}+V(\hat{x})
$$

where $\hat{p}$ is the Linear Momentum operator, $\hat{p}=-i \hbar \frac{\partial}{\partial x}$, and $\hat{x}$ is the position operator. $\hat{p}$ and $\hat{x}$ are both observables and thus are hermitian operators on the Hilbert Space, therefore $\hat{H}$ has a purely discrete spectrum of eigenvalues, $E_{n}$, that has a complete set of eigenstates, $\left|E_{n}(x)\right\rangle$, i.e. $\left(\hat{H}\left|E_{n}(x)\right\rangle=E_{n}\left|E_{n}(x)\right\rangle\right)$. Hence, any state or ket-vector, $|\Psi(x)\rangle$, can be expanded by the set of eigenstates, $|\Psi(x)\rangle=\sum_{n} a_{n}\left|E_{n}(x)\right\rangle$. Due to Schrodinger's equation the eigenstates pertaining to the energy operator are stationary with respect to time. For a diatomic molecule the oscillating like characteristics between the 2 atoms can be simply represented by a spring constant $K$. Given a particular natural frequency, $f_{e}, K$ can be represented as $K=4 \pi^{2} f_{e}^{2} m$. The potential energy pertaining to the simple oscillator is represented as

$$
\Phi(x)=-\int_{0}^{\infty} F d x=\int_{0}^{\infty} K x d x=\int_{0}^{\infty} 4 \pi^{2} f_{e}^{2} m x d x=2 \pi^{2} f_{e}^{2} m x^{2}
$$

Therefore the potential operator becomes $\hat{V}(\hat{x})=\frac{1}{2} m \omega^{2} \hat{x}^{2}$. Hamilton's operator can be pseudo separated as follows

$$
\begin{aligned}
\hat{a} & =\sqrt{\frac{m}{2}} \omega \hat{x}+\frac{i}{\sqrt{2 m}} \hat{p}=\frac{\sqrt{\hbar \omega}}{2}\left[(\hbar / m \omega)^{1 / 2} \frac{\partial}{\partial x}+(m \omega / \hbar)^{1 / 2} \hat{x}\right] \\
& =\frac{\sqrt{\hbar \omega}}{2}\left(\frac{\partial}{\partial \eta}+\hat{\eta}\right) \\
\hat{a}^{*} & =\sqrt{\frac{m}{2}} \omega \hat{x}-\frac{i}{\sqrt{2 m}} \hat{p}=\frac{\sqrt{\hbar \omega}}{2}\left[-(\hbar / m \omega)^{1 / 2} \frac{\partial}{\partial x}+(m \omega / \hbar)^{1 / 2} \hat{x}\right] \\
& =\frac{\sqrt{\hbar \omega}}{2}\left(-\frac{\partial}{\partial \eta}+\hat{\eta}\right)
\end{aligned}
$$

where $\hat{\eta}=(m \omega / \hbar)^{1 / 2} \hat{x}$. It can be shown that the commutator $\left[\hat{a}, \hat{a}^{*}\right]=\hbar \omega$ and therefore

$$
\hat{H}=\hat{a}^{*} \hat{a}+\frac{1}{2} \hbar \omega=\hat{a} \hat{a}^{*}-\frac{1}{2} \hbar \omega
$$

Based on Equation (20c) the eigenvalue equation can be written as

$$
\hat{a}^{*} \hat{a}\left|E_{n}(x)\right\rangle=\left(E_{n}-\frac{1}{2} \hbar \omega\right)\left|E_{n}(x)\right\rangle, \hat{a} \hat{a}^{*}\left|E_{n}(x)\right\rangle=\left(E_{n}+\frac{1}{2} \hbar \omega\right)\left|E_{n}(x)\right\rangle
$$

Built on the ideas in Equation (21) the concept of annihilators, $\hat{a}$, and creators, $\hat{a}^{*}$, are established; the following relationships exists 
$\hat{a}\left|E_{n}(x)\right\rangle=\left|E_{n-1}(x)\right\rangle$ corresponding eigenvalue $\left.E_{n-1}=E_{n}-\hbar \omega\right\}$ annihilator

$\hat{a}^{*}\left|E_{n}(x)\right\rangle=\left|E_{n+1}(x)\right\rangle$ corresponding eigenvalue $\left.E_{n+1}=E_{n}+\hbar \omega\right\}$ creator

Another possible solution, tied to the annihilator equation, is that

$\hat{a}\left|E_{0}(x)\right\rangle=0$. Here, $\left|E_{0}(x)\right\rangle$ is known as the zero-point wave-function and $E_{0}$ is the zero-point energy level. Equation (20a) pertaining to the zero-point state results in

$$
\begin{aligned}
& \quad \frac{\sqrt{\hbar \omega}}{2}\left(\frac{\partial}{\partial \eta}+\hat{\eta}\right) \psi_{0}=0, \therefore \frac{\partial \psi_{0}}{\partial \eta}+\hat{\eta} \psi_{0}=0 \text { normalized solution } \\
& \psi_{0}=\left(\frac{m \omega}{\pi \hbar}\right)^{1 / 4} e^{-\eta^{2} / 2}
\end{aligned}
$$

The eigenstates pertaining to the higher energy levels can be generated by applying the Riesz representation notation.

$$
\begin{aligned}
\left\langle\psi_{n+1} \mid \psi_{n+1}\right\rangle & =\left\langle\hat{a}^{*} \psi_{n} \mid \hat{a}^{*} \psi_{n}\right\rangle=\left\langle\psi_{n} \mid \hat{a} \hat{a}^{*} \psi_{n}\right\rangle=\left\langle\psi_{n} \mid\left(\hat{H}+\frac{\hbar \omega}{2}\right) \psi_{n}\right\rangle \\
& =\hbar \omega(n+1)\left\langle\psi_{n} \mid \psi_{n}\right\rangle
\end{aligned}
$$

Thus, if $\psi_{n}$ is normalized so is

$$
\psi_{n+1}=(\hbar \omega(n+1))^{-1 / 2} \hat{a}^{*} \psi_{n}=(n+1)^{-1 / 2} \frac{1}{2}\left(-\frac{\partial}{\partial \eta}+\hat{\eta}\right) \psi_{n}
$$

As a consequence, the recurring formula for the eigenfunctions/states becomes

$$
\psi_{n}=(2 n !)^{-1 / 2}\left(-\frac{d}{d \eta}+\eta\right)^{n} e^{-\frac{\eta^{2}}{2}}=(2 n !)^{-\frac{1}{2}}\left(\frac{m \omega}{\pi \hbar}\right)^{\frac{1}{4}} H_{n}(\eta) e^{-\frac{\eta^{2}}{2}}, n=0,1,2, \ldots
$$

where $H_{n}(\eta)$ are the Hermite polynomials. Additionally due to Equation (19a) the pertaining eigenvalues of $\hat{H}$ are $E_{n}=\hbar \omega\left(n+\frac{1}{2}\right)$

An alternative approach is to apply Schrodinger's Equation $i \hbar \frac{\partial \Psi}{\partial t}=\hat{H} \Psi(r, t)$.

Plugging in the definition for the Hamilton Operator defined in Equation (19a) results in the following simple oscillator equation

$$
\frac{d^{2} \Psi_{v}}{d x^{2}}+\frac{2 m}{\hbar^{2}}\left(E_{j}-\Phi(x)\right) \Psi_{v}=\frac{d^{2} \Psi_{v}}{d x^{2}}+\frac{2 m}{\hbar^{2}}\left(E_{j}-\frac{1}{2} m(\omega x)^{2}\right) \Psi_{v}=0
$$

Setting

$$
z=\sqrt{\frac{m \omega}{\hbar}} x, \alpha=\frac{2 m E_{j}}{\hbar^{2}}, \text { and } \Psi_{v}=e^{\frac{-z^{2}}{2}} w
$$

Schrodinger's Equation simplifies to

$$
\frac{d^{2} w}{d z^{2}}-2 z \frac{d w}{d z}+\left(\frac{\alpha}{\left(\frac{d z}{d x}\right)^{2}}-1\right) w=\frac{d^{2} w}{d z^{2}}-2 z \frac{d w}{d z}+\left(\frac{2 E_{j}}{\hbar \omega}-1\right) w=0
$$


When $\frac{2 E_{j}}{\hbar \omega}-1=2 j=0,1,2,3, \ldots$ the solutions to this equation are the Hermite Polynomials. Note that the solution is equivalent to Equation (22). Applying this definition and solving for $E_{j}$, the energy levels are defined as

$$
E_{j}=\hbar \omega\left(j+\frac{1}{2}\right)=j=0,1,2, \ldots
$$

Due to the assumption of independence of the different energies the partition function pertaining to internal energies can be represented as

$$
Z_{\text {int }}=Z_{r o t} Z_{v} Z_{\text {chem }}
$$

The partition function for the vibrational portion pertaining to a particular type of molecule $\mathrm{v}$ (e.g. $\mathrm{O}_{2}, \mathrm{~N}_{2}$ ) is represented as

$$
Z_{v}=\sum_{v_{j}} e^{-\left[\frac{\left(\epsilon_{v_{j}}-\epsilon_{v_{0}}\right)}{k T_{v}}\right]} \quad \beta=\frac{1}{\overline{\bar{T}}}=\frac{1}{k T_{v}}
$$

$\epsilon_{v_{0}}$ is the ground vibrational state energy (obtained by setting $j=0$ in Equation (26)). Using Equation (26) in (28), the vibrational partition function can be rewritten as

$$
Z_{v}=\sum_{j=0}^{\infty} e^{-\left[\frac{\hbar \omega}{k T_{v}}\right] j}=\frac{1}{1-e^{-\left[\frac{\hbar \omega}{k T_{v}}\right]}}=\frac{1}{1-e^{-\frac{\theta_{e}^{*}}{T_{v}}}}
$$

Such that $\theta_{e}^{*}=\frac{\hbar \omega}{k}$.

Applying Equation (6), an expression for the average energy is obtained

$$
u_{\text {int }}=R T^{2}\left(\frac{\partial \ln Z_{\text {int }}}{\partial T}\right)_{V}
$$

Therefore the vibrational internal energy becomes

$$
\left(u_{v}-u_{v_{0}}\right)=-R T_{v}^{2}\left(\frac{e^{-\frac{\theta_{e}^{*}}{T_{v}}}}{1-e^{-\frac{\theta_{e}^{*}}{T_{v}}}}\left(-\frac{\theta_{e}^{*}}{T_{v}^{2}}\right)\right)=\frac{R \theta_{e}^{*}}{e^{\frac{\theta_{e}^{*}}{T_{v}}}-1}
$$

Differentiating this equation with respect to temperature one obtains [7] [8]

$$
\frac{d u_{v}}{d T_{v}}=C_{v_{v}}=\frac{\theta_{e}^{* 2}}{T_{v}^{2}} \frac{\operatorname{Re}^{\frac{\theta_{e}^{*}}{T_{v}}}}{\left(e^{\frac{\theta_{e}^{*}}{T_{v}}}-1\right)^{2}}
$$

Applying the Boltzmann distribution definition for entropy

$$
s=-\sum_{i} p_{i} \ln \left(p_{i}\right)=\beta E+\ln (Z(\beta))
$$

results in 


$$
S_{v}=R\left(-\frac{\ln \left(1-e^{-\frac{\theta_{e}^{*}}{T_{v}}}\right)}{R}+\frac{\frac{\theta_{e}^{*}}{k T_{v}}}{e^{\frac{\theta_{e}^{*}}{T_{v}}}-1}\right)
$$

Recall that $T_{v} \ll T_{t r}, T_{r o t}$ and the temperatures pertaining to Translational and rotational energy levels are high enough to be considered by classical mechanics. The combination of kinetic energies $\left(\frac{p^{2}}{2 m}+\frac{L^{2}}{2 I}\right)$ and positions are considered as possible states, the application of the Boltzmann distribution results in the following equation for average energies.

$$
-\sigma_{n}=p-\mu_{B} \nabla \cdot \mathbf{v} \text { such that } \mu_{B}=\frac{p}{\beta_{r o t} N_{c}} \frac{u_{r o t}^{2}}{u^{2}}=\text { bulk viscosity }
$$

The instantaneous change of entropy can be represented in the following form

$$
T d s=d u+p d \rho^{-1}+\sum_{v} A_{v} d T_{v}
$$

The $\sum_{v} A_{v} d T_{v}$ terms represent the small deviation from equilibrium. The affinities $A_{v}$ are to be determined. The portion devoid of vibration energy is denoted from now on with subscript fr (frozen), and the portion pertaining to vibrational energy is indicated with subscript $\mathrm{v}$ [9].

$$
\begin{gathered}
s=s_{f r}\left(u_{t r}+u_{r o t}, \rho^{-1}\right)+\sum_{v} s_{v}\left(T_{v}\right) \\
T d s_{f r}=d\left(u_{t r}+u_{r o t}\right)+p d \rho^{-1}
\end{gathered}
$$

Set

$$
\begin{aligned}
& u_{t r}+u_{r o t}=C_{V, f r} T \text { assuming ideal gas } P=\rho R T \\
& C_{V, f r}=\frac{R}{\gamma-1}, C_{V, f r}=\text { coefficient of specific heat at constant volume. }
\end{aligned}
$$

Substituting Equation (38) into (37) and solve for $s_{f r}$ the following expression is obtained

$$
s_{f r}=C_{V, f r} \ln \left(u-\sum_{v} u_{v}\right)+R \ln \rho^{-1}+\text { const recall that } u-\sum_{v} u_{v}=u_{t r}+u_{r o t}
$$

Utilizing Equation (37) - (40) and applying them into Equation (35) yields

$$
A_{v}=T\left(\frac{-C_{V, f r}}{u_{t r}+u_{r o t}} \frac{d u_{v}}{d T_{v}}+\frac{1}{T_{v}} \frac{d u_{v}}{d T_{v}}\right)=\left(\frac{T}{T_{v}}-1\right) \frac{d u_{v}}{d T_{v}}=\left(\frac{T}{T_{v}}-1\right) C_{V, v}
$$

The expression in Equation (32) can be substituted into Equation (41). Since $T_{\nu} \ll \theta_{e}^{*}, C_{V, v}$ can deduce to

$$
C_{V, v}=\frac{d u_{v}}{d T_{v}}=\frac{n_{v}}{n} R\left(\frac{\theta_{e}^{*}}{T_{v}}\right)^{2} e^{-\frac{\theta_{e}^{*}}{T_{v}}}
$$

$C_{V, v}$ act as the specific heat pertaining to each molecular type v.

The inclusion of these energy terms into the standard energy equations results 
in

$$
\frac{D u}{D t}-\sigma_{n} \frac{D \rho^{-1}}{D t}=T \frac{D s_{v}}{D t}-\sum_{v} s_{v} \frac{D T_{v}}{D t}-\mu_{B} \nabla \cdot \mathbf{v} \frac{D \rho^{-1}}{D t}
$$

Applying the conservation of mass and the heat flux the entropy balance equations become

$$
\rho \frac{D s}{D t}+\nabla \cdot \frac{\mathbf{q}}{T}=\frac{1}{T} \mu_{B}(\nabla \cdot \mathbf{v})^{2}+\frac{1}{2 T} \mu \overrightarrow{\mathbf{D} \nabla v}: \overrightarrow{D \nabla v}+\frac{\kappa}{T^{2}}(\nabla T)^{2}+\frac{\rho}{T} \sum_{v} A_{v} \frac{D T_{v}}{D t}
$$

The Navier Stokes Equation with the inclusion of the bulk viscosity becomes

$$
\rho \frac{D \mathbf{v}}{D t}=-\nabla p+\nabla\left(\mu_{B} \nabla \cdot \mathbf{v}\right)+\mu \nabla \cdot \overrightarrow{D \nabla v}
$$

Applying conservation of energy results in the following expression for the vibration energy

$$
\frac{D u_{v}}{D t}=C_{V, v} \frac{D T_{v}}{D t}=n_{v} N_{c v} \Delta \epsilon_{v}
$$

$N_{c v}$ represents the number of collisions a molecule of type $v$ has per unit time, $n_{v}$ is the number of molecules of type $v$, and $\Delta \epsilon_{v}$ is the average energy gained per collision. It is known that most of the high temperature resides in the translational and rotational portions, therefore $\Delta \epsilon_{v}$ can best be expressed as

$$
\Delta \epsilon_{v}=\beta_{v} k\left(T-T_{v}\right)
$$

and yields

$$
\frac{D T_{v}}{D t}=\left(T-T_{v}\right) \frac{1}{\tau_{v}} \text { such that } \tau_{v}=\frac{C_{V, v}}{n_{v} N_{c v} k \beta_{v}}
$$

More elaborate modeling for $\Delta \epsilon_{v}$ can be achieved by applying Discrete Boltzmann Equations but these are outside the scope of this project [10]. Solving the differential equation for $T_{v}$ results in

$$
T_{v}=T-\left(T-T_{v}(0)\right) e^{-t / \tau_{v}}
$$

If $T$ is changed by an amount $\Delta T$ it will take a time of $\tau_{v}$ for the incremental change in $T_{v}$ is $\left(1-e^{-1}\right) \Delta t$. Therefore $\tau_{v}$ is called the relaxation time. And it is this relaxation time that introduces the hysteretic energy losses [11].

The energy conservation dissipation equation is in the form of

$$
\frac{\partial w}{\partial t}+\nabla \cdot \boldsymbol{I}=-D
$$

where

$$
\begin{gathered}
w=\frac{1}{2} \rho_{o} v^{2}+\frac{1}{2} \frac{p^{2}}{\rho_{o} c^{2}}+\frac{1}{2}\left(\frac{\rho T}{C_{p}}\right)_{o} s_{f r}^{2}+\sum_{v} \frac{1}{2}\left(\frac{\rho C_{V, v}}{T}\right)_{o} T_{v}^{2} \\
\boldsymbol{I}=p \mathbf{v}-\mu_{B} \mathbf{v}(\nabla \cdot \mathbf{v})-\mu \mathbf{v} \cdot(\overrightarrow{\boldsymbol{D} \nabla v})-\kappa T_{0}^{-1} T \nabla T \\
D=\mu_{B}(\nabla \cdot \mathbf{v})^{2}+\frac{1}{2} \mu(\overrightarrow{\boldsymbol{D} \nabla v}):(\overrightarrow{\boldsymbol{D} \nabla v})+\kappa T_{0}^{-1}(\nabla T)^{2} \\
+\sum_{v}\left(\frac{\rho_{o} C_{V, v}}{T \tau_{v}}\right)_{o}\left(T-T_{v}\right)^{2}
\end{gathered}
$$


From the coupled system of acoustic equation, asymptotic techniques result in different dispersion relationships for 3 different regimes; for the viscous and entropy boundary layers, and the equations pertaining to the acoustic mode. These translate to different set of partial differential equations pertaining to the 3 different regimes. The vibrational relaxation terms will mostly affect the acoustic mode regime. In order to obtain the new dispersion relationship a solution in the form of $e^{i k x} e^{-i \omega t}$ is plugged into the equation of motion (see Appendix A.1 - A.5), the ensuing is obtained

$$
\begin{aligned}
k^{2} \hat{p} & =\left(\omega^{2}+i \omega k^{2} \frac{\mu_{B}+\frac{4}{3} \mu}{\rho_{0}}\right) \hat{\rho} \\
i \omega \rho_{0} \hat{s}_{f r}= & {\left[\left(\frac{\kappa}{T}\right)_{o} k^{2}-\left(\frac{i \omega \rho}{T}\right)_{0} \sum_{v} \frac{C_{V, v}}{1-i \omega \tau_{v}}\right] \hat{T} } \\
\hat{\rho} & =\frac{\hat{p}}{c^{2}}-\frac{\left(\beta T / C_{p}\right)_{0}^{2}\left(\hat{s}_{f r} / \hat{T}\right) \hat{p}}{1-\left(T / C_{P}\right)_{0}\left(\hat{s}_{f r} / \hat{T}\right)}
\end{aligned}
$$

Plugging this last equation into Equation (51a) the dispersion relation is obtained [12]

$$
\frac{k^{2}}{\omega^{2}+i \omega k^{2} \frac{\mu_{B}+\frac{4}{3} \mu}{\rho_{0}}}=\frac{1}{c^{2}}-\frac{(\gamma-1) T_{0}\left(\hat{s}_{f r} / T\right) / C_{p} c^{2}}{1-\left(T / C_{p}\right)_{0}\left(\hat{s}_{f r} / \hat{T}\right)}
$$

For the acoustic mode and the frequency range of interest the following approximations exist: $k^{2}=\left(\frac{\omega}{c}\right)^{2}, \frac{C_{V, v}}{C_{p}} \ll 1, \frac{\omega \kappa}{\rho_{0} C_{p} c^{2}} \ll 1, \frac{\omega \mu}{\rho_{0} c^{2}} \ll 1$ the last 2 expressions can be found in Kirchhoff s dispersion relation and were considered small in [14]. Given these assumptions, the following asymptotic expansion is obtained

$$
k=\frac{\omega}{c}+i \frac{\omega^{2} \mu}{2 \rho_{0} c^{3}}\left[\frac{4}{3}+\frac{\mu_{B}}{\mu}+\frac{(\gamma-1) \kappa}{C_{p} \mu}\right]+\frac{1}{2}(\gamma-1) \frac{\omega}{c} \sum_{v} \frac{C_{V, v} / C_{p}}{1-i \omega \tau_{v}}
$$

The absorption coefficients are the imaginary portion of Equation (51e)

$$
\frac{\omega^{2} \mu}{2 \rho_{0} c^{3}}\left[\frac{4}{3}+\frac{\mu_{B}}{\mu}+\frac{(\gamma-1) \kappa}{C_{p} \mu}\right]+\frac{1}{2}(\gamma-1) \frac{\omega^{2}}{c} \sum_{v} \frac{\tau_{v} C_{V, v} / C_{p}}{1+\left(\omega \tau_{v}\right)^{2}}
$$

where

$$
\alpha_{v}=\frac{\pi}{2 \lambda}(\gamma-1) \frac{\omega^{2}}{c} \frac{\tau_{v} C_{V, v} / C_{p}}{1+\left(\omega \tau_{v}\right)^{2}}
$$

Recall that $\lambda=\frac{2 \pi c}{\omega}$. At relatively low frequencies, $\omega \ll \frac{1}{\tau_{v}}$ Equation (51g) indicates that 


$$
\alpha_{v} \rightarrow \frac{\tau_{v}}{c} \frac{C_{V, v}}{C_{p}}(\gamma-1) \omega^{2} \text { quadratic increase w.r.t. } \omega
$$

and at relatively high frequencies, $\omega \gg \frac{1}{\tau_{v}}$, results in the following

$$
\alpha_{v} \rightarrow \frac{\tau_{v}}{c} \frac{C_{V, v}}{C_{p}}(\gamma-1) \text { approaches a constant value }
$$

\subsection{Energy Loss: Thermal Boundary Layer-Bessel Functions}

In [1], multi-scales technique is used on the coupled fluid/structure set of equations. Equation (16), in that publication, represents the partial differential equation that connects fluctuations of temperature and pressure in the fluid medium. The dimensional form of this equation is

$$
\nabla_{y}^{2} T_{0}+i \omega \rho_{0} \frac{P r}{v} T_{0}=\frac{i \omega P_{0}}{\kappa} \text { in } D_{f}
$$

Before deriving the thermal exchange for a general porous medium (with pores of arbitrary geometry), the thermal exchange for a typical cylindrical pore with cross-sectional radius $R$ will be analyzed. The form this solution takes will be used as a pattern to emulate when considering solutions for the general case. Equation (52) in cylindrical form is

$$
\frac{1}{r} \frac{d}{d r}\left(r \frac{d T_{0}}{d r}\right)+i \omega \rho_{0} \frac{P r}{v} T_{0}=\frac{i \omega P_{0}}{\kappa} \text { in } D_{f}
$$

The Bessel function generator is defined as

$$
e^{i x \sin (\theta)}=J_{0}(x)+i 2 J_{1}(x) \sin \theta+2 J_{2}(x) \cos 2 x+\ldots
$$

The $J_{0}$ Bessel function is efficiently represented via Hankel's integral

$$
J_{0}(x)=\frac{1}{\pi} \int_{-1}^{1} \frac{e^{i x t}}{\sqrt{\left(1-t^{2}\right)}} d t=\frac{2}{\pi} \int_{0}^{1} \frac{\cos (x t)}{\sqrt{\left(1-t^{2}\right)}} d t
$$

It can be deduced from Equation (54a) that

$$
\frac{2 n}{x} J_{n}(x)=J_{n-1}(x)+J_{n+1}(x) \text { and } 2 \frac{\partial J_{n}(x)}{\partial x}=J_{n-1}(x)-J_{n+1}(x)
$$

The ensuing 2 equations are obtained by combining the equations in (55a)

$$
J_{n-1}(x)=\frac{n}{x} J_{n}(x)+\frac{\partial J_{n}(x)}{\partial x} \text { and } J_{n+1}(x)=\frac{n}{x} J_{n}(x)-\frac{\partial J_{n}(x)}{\partial x}
$$

Applying Equation (55b) results in

$$
\frac{d}{d x}\left\{x J_{1}(x)\right\}=x J_{0}(x)
$$

Equation (55c) is integrated from 0 to $R$, this generates an equation that will be utilized shortly

$$
\int_{0}^{R} x J_{0}(\gamma x) d x=\frac{R}{\gamma} J_{1}(\gamma)
$$


Teagle [1] showed that $P_{0}$ is independent of the micro (fast) scale y. Therefore the particular solution for Equation (53) is $\frac{v}{\kappa P r} P_{0}=\frac{\rho_{0}}{C_{p}} P_{0}$. The homogeneous solution is $A_{1} J_{0}\left(\sqrt{\frac{i \omega \rho_{0} P r}{\mu} r}\right)$, where $A_{1}$ is a derived so that boundary conditions at the wall of the cylinder pores are met, $T_{0}(R)=0$. Combining the particular and homogenous solutions and satisfying boundary conditions, the following expression for temperature is obtained

$$
T_{0}(r)=\frac{\rho_{0}}{C_{p}} P_{0}\left(1-\frac{J_{0}(\chi r)}{J_{0}(\chi R)}\right) \ni \chi=\sqrt{\frac{i \omega \rho_{0} P r}{\mu}}
$$

For statistical energy analysis calculations, average temperature of the pore cross-section suffices, therefore

$$
\begin{aligned}
\left\langle T_{0}\right\rangle & =\frac{\int_{0}^{R} 2 \pi T_{0}(r) r d r}{\pi R^{2}}=\frac{\rho_{0}}{C_{p}} P_{0}\left(1-\frac{2 J_{1}(\chi R)}{R \chi J_{0}(\chi R)}\right) \\
& =\frac{\rho_{0}}{C_{p}} P_{0}\left(1-(1-i) \frac{2 J_{1}(\chi R)}{R J_{0}(\chi R)} \sqrt{\frac{\mu}{\omega \rho_{0} P r}}\right)
\end{aligned}
$$

For the more general case, the focus is on analyzing the energy flux escaping the acoustic mode into the entropy boundary layer. Johnson et al. [2] accomplished similar work for the viscous boundary layer.

Trilling [13] gave a detailed explanation of the separation technique utilized to obtain 3 dispersion relations from the Navier-Stokes Equation. Applying asymptotic expansion for each of these relationships, it is possible to derive 3 sets of simple partial differential equations along with their pertinent conditions. The process polarized the linear Navier-Stokes equation into 3 different flow regimes. The superposition principle can be applied to obtain the total velocity. The flow regimes or modes can be characterized by the following:

Vorticity Mode field

$$
\nabla^{2} \mathbf{v}_{v o r}=\frac{\rho}{\mu} \frac{\partial \mathbf{v}_{v o r}}{\partial t}, \nabla \cdot \mathbf{v}_{v o r}=0, \quad s_{v o r}=p_{v o r}=T_{v o r}=0
$$

It is important to point out that Equation (58) is a classical diffusion equation The entropy Mode field

$$
\begin{gathered}
\nabla^{2} s_{e n t}=\frac{\rho c_{P}}{\kappa} \frac{\partial s_{e n t}}{\partial t} \\
p_{e n t}=0, \mathbf{v}_{e n t}=\left(\frac{\beta T \kappa}{\rho c_{P}}\right)_{o} \nabla s_{e n t}, \nabla \times \mathbf{v}_{e n t}=0, \\
T_{e n t}^{\prime}=\left(\frac{T}{c_{P}}\right) s_{e n t}, \rho_{e n t}^{\prime}=\left(\frac{\beta T \rho}{c_{P}}\right)_{o} s_{e n t}
\end{gathered}
$$

Acoustic Mode field

$$
\rho \frac{\partial \mathbf{v}_{a c}}{\partial t}=-\nabla p_{a c}, s_{a c}=0, \nabla \times \mathbf{v}_{a c}=0, T_{a c}^{\prime}=\left(\frac{\beta T}{\rho c_{P}}\right)_{o} p_{a c}, \rho_{a c}^{\prime}=\frac{p_{a c}}{c^{2}}
$$


Due to the superposition principle the microscopic velocity flow field can be written as

$$
\mathbf{v}=\mathbf{v}_{v o r}+\mathbf{v}_{e n t}+\mathbf{v}_{a c}
$$

The dispersion for the vorticity and entropy modes are

$$
k_{v o r}^{2}=\frac{i \omega \rho}{\mu} \text { and } k_{\text {ent }}^{2}=\frac{i \omega \rho c_{P}}{\kappa}
$$

Therefore the vorticity and entropy mode field extend to $\frac{1}{\left|k_{v o r}\right|}$ and $\frac{1}{\left|k_{\text {ent }}\right|}$ so

$$
\delta_{v o r}=\left(\frac{2 \mu}{\omega \rho}\right)^{1 / 2} \text { and } \delta_{\text {ent }}=\left(\frac{2 \kappa}{\omega \rho c_{P}}\right)^{1 / 2}=\frac{\delta_{v o r}}{(P r)^{1 / 2}}
$$

Near the solid surface it can be assumed that the solution has the form

$$
\Psi(x, y, z)=\Psi(x, y, 0) e^{-(1-i) z / \delta_{\text {ent }}} .
$$

where this relation satisfies the following equation

$$
\frac{\partial^{2} \Psi(x, y, z)}{\partial z}=\frac{-i 2}{\delta^{2}} \Psi(x, y, z) \text {. Additionally, as in the aforementioned }
$$

cylindrical case, the temperature fluctuation at the wall is zero. The temperature conditions in Equations (58) (29b) (60) produces the following

$$
T_{a c}^{\prime}+T_{e n t}=0
$$

Applying Equation (64) into Equations (58), (59a), (59b) and splitting the "del" operator into tangential and normal components (where the tangential portion will have a subscript $\mathrm{T}$ ) yields the following

$$
\begin{gathered}
\nabla_{T} \cdot \mathbf{v}_{v o r, T}(\mathbf{x}, \mathbf{y})-(1-i) \mathbf{v}_{v o r}(\mathbf{x}, \mathbf{y}) \cdot \frac{\mathbf{n}}{\delta_{v o r}}=0 \\
\mathbf{v}_{e n t, T}(\boldsymbol{x}, \boldsymbol{y})=\frac{\beta \kappa}{\rho c_{P}} \nabla_{T} T_{e n t}=0 \\
\mathbf{v}_{e n t} \cdot \boldsymbol{n}=-\frac{\beta \kappa}{\rho c_{P}}(1-i) \frac{T_{e n t}}{\delta_{\text {ent }}}
\end{gathered}
$$

At $z=0$ plane Equation (61) will give the following boundary condition

$$
\mathbf{v}_{\text {wall }}=\mathbf{v}_{\text {vor }}+\mathbf{v}_{\text {ent }}+\mathbf{v}_{a c}
$$

and applying the $\nabla_{T}$. (horizontal divergence) along with Equation (67), Equation (69) can be rewritten the following way

$$
\nabla_{T} \cdot \mathbf{v}_{a c, T}(\mathbf{x}, \mathbf{y})+(1-i) \mathbf{v}_{v o r}(\mathbf{x}, \mathbf{y}) \cdot \frac{\mathbf{n}}{\delta_{v o r}}=0
$$

Remember that

$$
\begin{aligned}
\gamma-1 & =\frac{T \tilde{\beta}^{2} \gamma K_{T}}{\rho C_{p}}=\frac{T \tilde{\beta}^{2} K_{S}}{\rho C_{p}}=\frac{T \tilde{\beta}^{2} c^{2}}{C_{p}} K_{T} / K_{S} \\
& =\text { isothermal/adiabatic bulk modul }
\end{aligned}
$$

Additionally, using Equation (68) and (70) the subsequent conditions are obtained in the direction normal to the surface 


$$
\mathbf{v}_{\text {wall }} \cdot \mathbf{n}=-(1+i) \frac{\delta_{v o r}}{2} \nabla_{T} \cdot \mathbf{v}_{a c, T}(\mathbf{x}, \mathbf{y})+(1-i)(\gamma-1) \frac{\omega}{c^{2}} \frac{\delta_{e n t}}{2} \frac{p_{a c}}{\rho}+\mathbf{v}_{a c} \cdot \mathbf{n}
$$

Recall that the main goal is to obtain an expression for the energy that is being dissipated and this is dictated by the net power flowing out of the acoustic (ac) mode close to the boundary. Multiplying Equation (72) by the complex conjugate of the acoustic mode pressure, $p_{a c}^{*}$, and solving for $-p_{a c}^{*} \mathbf{v}_{a c} \cdot \mathbf{n}$, which is the intensity leaving the acoustic mode, provides the following equation

$$
\begin{aligned}
& -\left(\boldsymbol{I}_{a c} \cdot \boldsymbol{n}\right)_{a v} \\
& =\frac{1}{2} \operatorname{Re}\left(p_{a c}^{*} \mathbf{v}_{\text {wall }} \cdot \mathbf{n}\right)-\frac{\delta_{v o r}}{4} \nabla \cdot\left\{\operatorname{Re}\left[(1+i) p_{a c}^{*} \mathbf{v}_{a c, T}(\mathbf{x}, \mathbf{y})\right]\right\} \\
& +\frac{\delta_{v o r}}{4} \operatorname{Re}\left[(1+i) \nabla_{T} p_{a c}^{*} \cdot \mathbf{v}_{a c, T}(\mathbf{x}, \mathbf{y})\right] \\
& +\operatorname{Re}\left[\frac{1}{2}(1-i)(\gamma-1) \frac{\omega}{c^{2}} \frac{\delta_{e n t}}{2} \frac{\left|p_{a c}\right|^{2}}{\rho}\right]
\end{aligned}
$$

Recall that the relative velocity at the wall is equal to zero. From the first relation in Equation (60) it can be deduced that

$$
\nabla_{T} p_{a c}^{*}=i \omega \rho \mathbf{v}_{a c, T}^{*}(\mathbf{x}, \mathbf{y})
$$

The second term in the right hand side of Equation (73) will average out to zero given enough surface area. Equation (74) will be applied in the third term of Equation (73) to finally obtain the following relation

$$
\left(\frac{d^{2} E}{d A d t}\right)_{d i s s}=\left(\frac{\omega \rho \mu}{2}\right)^{1 / 2}\left(\mathrm{v}_{a c, T}^{2}(\mathbf{x}, \mathbf{y})\right)_{a v}+(\gamma-1)\left(\frac{\omega \rho \kappa}{2 c_{P}}\right)^{1 / 2} \frac{\left|p_{a c}\right|_{a v}^{2}}{\rho c^{2}}
$$

For the mechanical dissipation the first term in the right hand side is used by [2]. The second term will be utilized in this study to treat the thermal dissipation and derive the acoustic bulk modulus.

The thermal counterpart of dynamic permeability and tortuosity are

$$
\begin{gathered}
\phi T_{o}=-\frac{k^{\prime}(\omega)}{\kappa} \frac{\partial P_{o}}{\partial t} \\
\alpha^{\prime}(\omega) c_{P} \rho_{f} \frac{\partial T_{o}}{\partial t}=\frac{\partial P_{o}}{\partial t}
\end{gathered}
$$

where $T_{o}$ and $P_{o}$ satisfy the following

$$
c_{P} \rho_{f} \frac{\partial T_{o}}{\partial t}=\kappa \nabla^{2} T_{o}+\frac{\partial P_{o}}{\partial t} \nabla P_{o}=0 \text { in } \boldsymbol{r} \in V \text { and } T_{o}=0 \quad \boldsymbol{r} \in \partial V
$$

The real constant $k_{o}^{\prime}=\lim _{\omega \rightarrow 0} k^{\prime}(\omega)$ acts like a thermal form of a static thermal permeability, i.e. the thermal counterpart to Darcy's static equation of $k_{o}$.

A volume of porous sample enclosed by a thin membrane is considered. In this particular case, it is assumed that the thin membrane acts like an adiabatic wall. The interest is to know where the singularities to $k^{\prime}(\omega)$ and $\alpha^{\prime}(\omega)$ lie. The existence of a singularity means that there exists a nontrivial solution to the above microscopic equation while the macroscopic input is zero, i.e. $P_{o}=0$. 
This is equivalent to saying that there is no mechanical power entering the element. Therefore Equation (78) becomes

$$
c_{P} \rho_{f} \frac{\partial T_{o}}{\partial t}=\kappa \nabla^{2} T_{o}
$$

To guarantee that no thermal power is entering the element, the following has to hold

$$
\iint T_{0}^{*}(\boldsymbol{x}, \boldsymbol{y}) \nabla_{y} T_{o}(\boldsymbol{x}, \boldsymbol{y}) \cdot \boldsymbol{n} d A_{y}=0
$$

Since the membrane is adiabatic and since conditions listed in Equation (78) are being satisfied the integral in Equation (80) is consequentially fulfilled. Pre multiplying the Fourier equation of heat diffusion equation, Equation (79), by the complex conjugate $T_{0}^{*}$, assuming a solution of the form $e^{-i \omega t}$, and then perform a volume integral in terms of the microscopic variable yields

$$
-i \omega c_{P} \rho_{f} \iiint T_{0}^{*} T_{o} d V_{y}=\kappa \iiint T_{0}^{*} \nabla^{2} T_{o} d V_{y}
$$

Making use of the usual identification

$$
\begin{aligned}
& \nabla \cdot\left(T_{0}^{*} \nabla T_{o}\right)=\nabla T_{0}^{*} \cdot \nabla T_{o}+T_{0}^{*} \nabla^{2} T_{o} \\
\therefore & T_{0}^{*} \nabla^{2} T_{o}=\nabla \cdot\left(T_{0}^{*} \nabla T_{o}\right)-\nabla T_{0}^{*} \cdot \nabla T_{o}
\end{aligned}
$$

So

$$
\begin{aligned}
& \iiint T_{0}^{*} \nabla^{2} T_{o} d V_{y}=\iiint\left(\nabla \cdot\left(T_{0}^{*} \nabla T_{o}\right)-\nabla T_{0}^{*} \cdot \nabla T_{o}\right) d V_{y} \\
& =\iint T_{0}^{*} \nabla T_{o} \cdot \boldsymbol{n} d A_{y}-\iiint\left|\nabla T_{o}\right|^{2} d V_{y}
\end{aligned}
$$

The first term in the right hand side of Equation (84) goes to zero because of boundary condition found in Equation (80). Inserting this result in Equation (81) and solving for $\omega$ the following is obtained

$$
\omega=-i \frac{\kappa}{c_{P} \rho_{f}} \frac{\iiint\left|\nabla_{y} T_{o}\right|^{2} d V_{y}}{\iiint\left|T_{o}\right|^{2} d V_{y}} \text { in } D_{f}
$$

Due to the shifting rule, this indicates that the singularity for $k^{\prime}(\omega)$ lies in the negative imaginary axis. In the meantime consider Equation (77) where the boundary condition is that of $T_{o}=0$. Additionally, no mechanical power is entering through the adiabatic wall. The aim is to find where the singularities of $\alpha^{\prime}(\omega)$ lie. Equation (78) is multiplied by $T_{0}^{*}$. A volume integral results in

$$
-i \omega c_{P} \rho_{f} \iiint T_{0}^{*} T_{o} d V_{y}=\kappa \iiint T_{0}^{*} \nabla^{2} T_{o} d V_{y}-i \omega \iiint T_{0}^{*} P_{o} d V_{y}
$$

In [1] it is shown that $P_{o}$ is independent of the micro scale variable and therefore it is considered as an external source of power for this thermal element. Applying Equation (82), (83), and the Green's theorem to the first term in Equation (86), the same result of Equation (85) is obtained; again, the singularities of $\alpha^{\prime}(\omega)$ also lie in the negative imaginary axis.

Following the same steps performed in [2] in deriving the relation for viscous length, the last term in the right hand side of Equation (73) 
$\frac{1}{2}(1-i)(\gamma-1)\left(\frac{\omega \rho \kappa}{2 c_{P}}\right)^{1 / 2} \frac{\left|p_{a c}\right|_{a v}^{2}}{\rho c^{2}}$ is studied. It is understood that this term represents the thermal portion of the energy flux exiting the main acoustic mode. The third term in the RHS of Equation (50a) is the thermal energy. Multiplying this term by the speed of sound, $c$, the energy flux entering the pore is attained. The ensuing equation represents the ratio of thermal energy that is leaving the acoustic mode to that of thermal energy that is entering the thermal element.

$$
\frac{\iint_{\text {wall }} \frac{1}{2}(1-i)(\gamma-1) \frac{\omega}{c^{2}} \frac{\delta_{\text {ent }}}{2} \frac{\left|p_{a c}\right|^{2}}{\rho} d s}{\iiint_{V o l} c \frac{1}{2}\left(\frac{\rho T}{C_{p}}\right)_{o} s_{f r}^{2} d v}
$$

Recall temperature condition $T_{a c}^{\prime}+T_{e n t}=0$ and from Equation (59b), $T_{e n t}^{\prime}=\left(\frac{T}{c_{P}}\right) s_{f r}$. Additionally from Equation (60), $T_{a c}^{\prime}=\left(\frac{\tilde{\beta} T}{\rho c_{P}}\right)_{o} p_{a c}$. Lastly, applying Equation (71), Equation (87) simplifies to

$$
\frac{\omega}{c}(1-i) \delta_{e n t} \frac{\iint_{\text {wall }}\left|p_{a c}\right|^{2} d s}{2 \iiint_{V o l}\left|p_{a c}\right|^{2} d v}=\frac{\omega}{c}(1-i) \delta_{\text {ent }} \frac{S}{2 V}=\frac{\omega}{c}(1-i) \frac{\delta_{e n t}}{\Lambda^{\prime}}
$$

In Equation (88), $p_{a c}=P_{0}$. The integration takes place at the micro level and it has been established that $P_{0}$ is independent of the micro scale variable, $y$, and thus the first equality sign in Equation (88). $\Lambda^{\prime}$ is the Thermal Length and is defined as $\frac{S}{2 V}$. Using Equation (57) as a pattern solution, the general solution for average temperature is defined as (assuming ideal gas conditions)

$$
\left\langle T_{0}\right\rangle=\frac{1}{\rho_{0} C_{p}} P_{0}\left(1-(1-i) \frac{\delta_{\text {ent }}}{\Lambda^{\prime}}\right)
$$

In Equation (89), as increases the thinner the entropy boundary layer, $\delta_{e n t}$, is and therefore the limit as $\omega \rightarrow \infty$ the acoustic mode condition (ideal gas) $\left\langle T_{0}\right\rangle=\frac{\rho_{0}}{C_{p}} P_{0}$ applies for the whole cross-section. This equation for $\left\langle T_{0}\right\rangle$ is plugged into Equation (3). The following expression for thermal permeability is obtained

$$
k^{\prime}(\omega)=\frac{\kappa \phi}{-i \omega} \frac{1}{\rho_{0} C_{p}}\left(1-(1-i) \frac{\delta_{e n t}}{\Lambda^{\prime}}\right)=\frac{i \kappa \phi}{\omega \rho_{0} C_{p}}\left(1-\left(\frac{-i \kappa}{\omega \rho C_{p}}\right)^{1 / 2} \frac{2}{\Lambda^{\prime}}\right)
$$

The second term in Equation (90) is obtained by applying the definition of $\delta_{\text {ent }}$. This term can asymptotically (as $\omega \rightarrow \infty$ )be represented as

$$
\frac{1}{\frac{-i \omega \rho C_{p}}{\kappa \phi}-\left(\frac{-i 4 C_{p} \rho \omega}{\Lambda^{\prime 2} \kappa^{2} \phi^{2}}\right)^{1 / 2}}=\frac{k_{0}}{\frac{-i \omega \rho C_{p} k_{0}}{\kappa \phi}-\left(\frac{-i 4 C_{p} \rho \omega k_{0}^{2}}{\Lambda^{\prime 2} \kappa^{2} \phi^{2}}\right)^{1 / 2}}
$$

where, $k_{0}$ is the thermal permeability as $\omega \rightarrow 0$. A combined asymptotic re- 
presentation that interpolates thermal permeability for the whole frequency range is represented as

$$
k^{\prime}(\omega)=\frac{k_{0}}{\frac{-i \omega \rho C_{p} k_{0}}{\kappa \phi}+\left(1-\frac{-i 4 C_{p} \rho \omega k_{0}^{2}}{\Lambda^{\prime 2} \kappa^{2} \phi^{2}}\right)^{1 / 2}}
$$

Finally, this is the term that is used in Equation (2) to obtain the compressibility factor of the fluid of the porous medium.

\section{Conclusions and Closing Remarks: Absorption}

Consider the acoustic medium near the wall of a tube (the $\mathrm{x}$ axis is taken to be along the tube). Set $\theta_{i}$ as the angle of incident and recall that the solution to the wave equation tangent to the tube wall will have the form of $f\left(t-\frac{\boldsymbol{x}_{T}}{c}\right)=f\left(t-\boldsymbol{n}_{i, T} \cdot \boldsymbol{x}\right)$ therefore $c \boldsymbol{\nabla}_{T} p=-\boldsymbol{n}_{i, T} \partial p / \partial t$. Also recognize that $\boldsymbol{n}_{i, T} \cdot \boldsymbol{n}_{i, T}=\sin ^{2}\left(\theta_{i}\right)$. With these definitions, the $\nabla_{T} \boldsymbol{p}_{a c}^{*} \cdot \mathbf{v}_{a c, T}$ term in Equation (73) can be represented as

$$
\nabla_{T} \boldsymbol{p}_{a c}^{*} \cdot \mathbf{v}_{a c, T}=-\frac{\sin ^{2}\left(\theta_{i}\right)}{\rho c^{2}} \frac{\partial p}{\partial t}
$$

An expression for the wall impedance can be given as

$$
\begin{aligned}
\frac{1}{Z_{\text {wall }}} & =-\frac{\mathbf{v}_{\text {wall }} \cdot \mathbf{n}}{p}=0.5\left((1-i) \frac{\omega}{\rho c^{2}}\left[\delta_{\text {vor }} \sin ^{2}\left(\theta_{i}\right)+(\gamma-1) \delta_{\text {ent }}\right]\right) \\
& =\frac{e^{-i \pi / 4}}{\rho c}\left(\frac{\omega \mu}{\rho c^{2}}\right)^{1 / 2}\left[\sin ^{2}\left(\theta_{i}\right)+\frac{\gamma-1}{(P r)^{1 / 2}}\right]
\end{aligned}
$$

The objective is to obtain a simple partial differential equation that incorporates the impedance effects of the viscous and thermal boundary layer. Following techniques introduced by Crandall et al. [15], The variational form of the Helmholtz equation is

$$
\delta \int_{x_{1}}^{x_{2}} \iint \frac{1}{2}\left[M p^{2}-(\nabla p)^{2}\right] d A d x+\int_{x_{1}}^{x_{2}} \oint \delta p \nabla p \cdot \boldsymbol{n}_{\text {wall }} d l d x=0
$$

The $d l$ is a segment of the perimeter of the tube and $M=\frac{\omega^{2}}{c^{2}}+\frac{i 2 \omega^{3} \delta_{c l}}{c^{4}}$

Applying the definition of impedance, the following is obtained

$$
\nabla p \cdot \boldsymbol{n}_{\text {wall }}=\frac{i \omega \rho}{Z} p
$$

and applying this to the variational Helmholtz Equation yields

$$
\delta \int_{x_{1}}^{x_{2}}\left\{\iint \frac{1}{2}\left[M p^{2}-(\nabla p)^{2}\right] d A+\frac{i \omega \rho}{2 Z} \oint p^{2} d l\right\} d x=0
$$

As a first order approximation, it is assumed that the parameters do not vary with respect to the cross-sectional area 


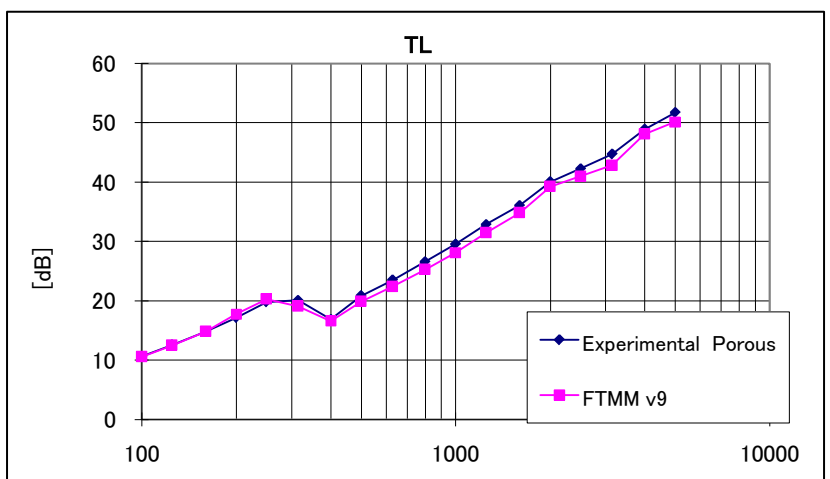

(a)

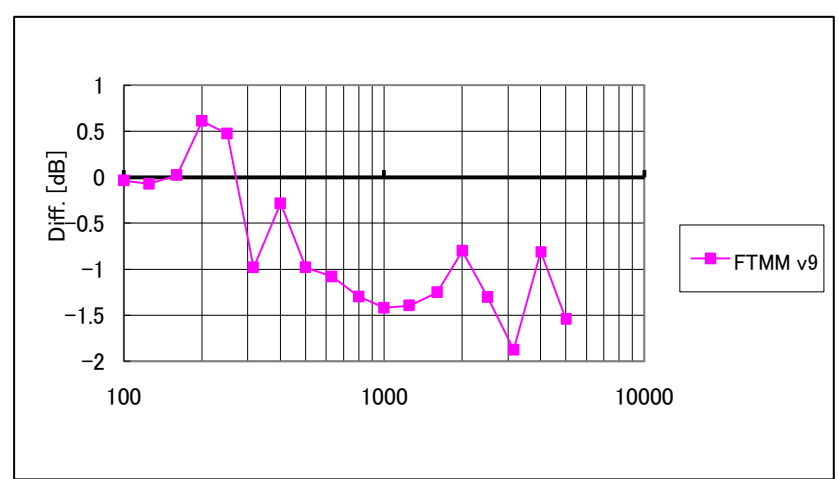

(b)

Figure 1. Left: TL comparison (dB); Right: TL difference(dB) measured data is the reference.

\section{\begin{tabular}{|l|l|l|l|}
\hline Case9 & Plate1_B* & Foam1_B & Foam2 \\
\hline
\end{tabular}}

Figure 2. Multi layer configuration.

$$
\delta \int_{x_{1}}^{x_{2}}\left\{\frac{1}{2}\left[M p^{2}-\left(\frac{\partial p}{\partial x}\right)^{2}\right] A+\frac{\omega \rho}{2 Z} p^{2} L_{p}\right\} d x=0 \quad L_{p} \text { perimeter length }
$$

Performing the variation generates

$$
\frac{d}{d x}\left(A \frac{d p}{d x}\right)+\left(M A+\frac{i \omega \rho}{Z} L_{p}\right) p=0
$$

For the case pertaining to constant cross-sectional area, the dispersion relationship is

$$
\begin{aligned}
& k^{2}=M+\frac{i \omega e^{-i \pi / 4}}{c}\left(\frac{\omega \mu}{\rho c^{2}}\right)^{1 / 2}\left[\sin ^{2}\left(\theta_{i}\right)+\frac{\gamma-1}{(P r)^{1 / 2}}\right] \frac{L_{p}}{A}=M+\frac{i \omega}{c}(1-i) \tilde{\alpha}_{\text {wall }} \\
& \tilde{\alpha}_{\text {wall }}=\left.e^{-i \pi / 4}\left(\frac{\omega \mu}{\rho c^{2}}\right)^{1 / 2}\left[\sin ^{2}\left(\theta_{i}\right)+\frac{\gamma-1}{(P r)^{1 / 2}}\right] \frac{L_{p}}{A}\right|_{\theta_{i}=\pi / 2} \text { is the absorption }
\end{aligned}
$$

coefficient of the wall. It is interesting to note the similarity in form between the imaginary part of Equations (100) and (88).

Equation (51g) pertaining to the absorption due to the relaxation process, $\alpha_{v}$, combined with the effect of the thermal permeability, $k^{\prime}(\omega)$, on the bulk modulus, $\beta$, of the porous medium are incorporated into Transmission Matrix Method with finite size correction via Green's functions techniques (FTMM) [16]. The parameters to this study are listed in Table 1 in [1]. Figure 1 shows the comparison between experimental data and the FTMM calculations for the multi-layer configuration shown in Figure 2. Further analysis, especially a parameter study on the different relaxation and thermal components is currently being done. This study mainly assumes air within the porous medium but there is current interest in applying these to pores with different rheological make up. 


\section{Conflicts of Interest}

The authors declare no conflicts of interest regarding the publication of this paper.

\section{References}

[1] Teagle-Hernandez, A., Ohtmer, O. and Nguyen, D. (2018) Mathematical Modeling of Porous Medium for Sound Absorption Simulations: Application of Multi-Scales and Homogenization. Journal of Applied Mathematics and Physics, 6, 2705-2717. https://doi.org/10.4236/jamp.2018.612224

[2] Johnson, D. and Koplik, J. (1987) Theory of Dynamic Permeability and Tortuosity in Fluid-Saturated Porous Media. Journal of Fluid Mechanics, 176, 379-402. https://doi.org/10.1017/S0022112087000727

[3] Thomasson, S.I. (1980) On the Absorption Coefficient. Acustica, 44, 265-273.

[4] Brouard, B., Lafarge, D. and Allard, J.F. (1995) A General Method of Modeling Sound Propagation in Layered Media. J. Sound Vib., 183, 129-142. https://doi.org/10.1006/jsvi.1995.0243

[5] Folds, D. and Loggins, C.D. (1977) Transmission and Reflection of Ultrasonic Waves in Layered Media. J. Acoust. Soc. Amer., 62, 1102-1109. https://doi.org/10.1121/1.381643

[6] Feynman, R. and Hibbs, A. (1965) Quantum Mechanics and Integrals, McGraw-Hill.

[7] ter Haar, D. (1954) Elements of Statistical Mechanics. Rinehart, New York. https://doi.org/10.1119/1.1933869

[8] Schiff, L.I. (1955) Quantum Mechanics. McGraw-Hill, New York.

[9] Landau, L. and Lifshitz, E. (1959) Fluid Mechanics. Pergamon.

[10] Bellomo, N. and Gatignol, R. Lecture Notes on the Discretization of the Boltzmann Equation. World Scientific.

[11] Herzfield, K.F. and Rice, F.O. (1928) Dispersion and Absorption of High Frequency Sound Waves. Phys. Rev., 31, 691-695. https://doi.org/10.1103/PhysRev.31.691

[12] Lighthill, M.J. (1956) Viscosity Effects n Sound Waves of Finite Amplitude, Batchelor and Davies (Eds.) Surveys in Mechanics. Cambridge University Press, London.

[13] Trilling, L. (1955) On Thermally Induced Sound Fields. J. Acoust. Soc. Am, 27, 425-431. https://doi.org/10.1121/1.1907920

[14] Truesdell, C. (1953) Precise Theory of the Absorption and Dispersion of Forced Plane Infinitesimal Waves According to the Navier-Stokes Equations. J. Ration. Mech. Anal., 2, 643-730. https://doi.org/10.1512/iumj.1953.2.52032

[15] Crandall, S.H., Karnopp, D.C., Kurtz Jr., E.F. and Pridmore-Brown, D.C. (1968) Dynamics of Mechanical and Electromechanical Systems. McGraw-Hill, New York.

[16] Nguyen, D. and Teagle-Hernadez, A. (2019) Application of Transfer Matrix Method, Green's Functions, and Variational Technique to Predict Diffuse Absorption Coefficient. CalTech-SOCAMS, Pasadena. 


\section{Appendix A: Linear Acoustic Equation}

$$
\begin{aligned}
& \frac{\partial \rho^{\prime}}{\partial t}+\rho_{0} \nabla \cdot v=0 \\
& \rho_{0} \frac{\partial v}{\partial t}=-\nabla p+\mu_{\beta} \nabla(\nabla \cdot v)+\mu \nabla \cdot(\overrightarrow{D \nabla v}) \\
& \rho_{0} \frac{\partial s_{f r}}{\partial t}+\sum_{\nu}\left(\frac{\rho}{T}\right)_{0} c_{v V} \frac{\partial T_{v}}{\partial t}-\frac{\kappa}{T_{0}} \nabla^{2} T^{\prime}=0 \\
& \rho^{\prime}=\frac{P}{c^{2}}-\left(\frac{\rho \beta T}{c_{p}}\right)_{0} s_{f r} \\
& T^{\prime}=\left(\frac{T \beta}{\rho c_{p}}\right)_{0} p+\left(\frac{T}{c_{p}}\right)_{0} s_{f r} \\
& \overrightarrow{D \nabla v}=\frac{1}{2}\left[\nabla v+(\nabla v)^{T}-\frac{2}{3} \vec{I} t r[\nabla v]\right] \\
& \nabla \stackrel{\text { Covariant }}{\underset{\text { Gradient }}{\longrightarrow}}(\nabla A)_{(i j)} \equiv \frac{1}{h_{(i)} h_{(j)}}\left(\partial_{i}\left(h_{(j)} A_{(j)}\right)-\sum_{k} \Gamma_{i j}^{k} h_{(k)} A_{(k)}\right) \\
& \Gamma_{i j}^{k}=\text { Christoffel, }(\nabla A)_{(i j)}=\text { physical tensor },(\nabla A)_{i j}=\text { covariant tensor } \\
& \text { metric tensor } g_{i j}=h_{(i)} h_{(j)} e_{i} \cdot e_{j}
\end{aligned}
$$




\section{Nomenclature}

$$
\begin{aligned}
& p_{0}=\text { Fluid Pressure, Macro Scale } \\
& \varphi=\text { Porosity } \\
& \eta=\text { Viscosity } \\
& D_{f}=\text { Domain Occupied by Fluid } \\
& \gamma=C_{p} / C_{v} \quad C_{p}=\text { specific heat at constant pressure } C_{V}=\text { Specific heat at con- }
\end{aligned}
$$
stant volume

$\kappa=$ Coefficient of thermal Conductivity

$\operatorname{Pr}=$ Prandlt Number $=\frac{\eta C_{p}}{\kappa}$

$\rho_{f}, \rho_{0}=$ density of the Fluid

$T=$ Temperature deviation

$T_{v}=$ Vibrational temperature pertaining to molecular type $\mathrm{v}$

$\bar{T}=$ theorist temperature, $k T, k=$ Boltzmann constant

$k(\omega)=$ Dynamic Viscous Permeability

$k^{\prime}(\omega)=$ Dynamic Thermal Permeability

$a^{*}=$ asterisk superscript means conjugate

$\vec{A}=$ special tilde ${ }^{-} 2^{\text {nd }}$ rank tensor

$s$ = Entropy, $s_{f r}=$ Entropy without molecular vibration,

$s_{v}=$ molecular vibration entropy

$\tilde{\beta}=$ Thermal expansion coefficient $=\rho^{-1}\left(\frac{\partial \rho}{\partial t}\right)_{p}$ 